\title{
Ethnologies
}

\section{Pierre Anctil et Ira Robinson (dirs.). Les communautés juives de Montréal, histoire et enjeux contemporains. (Québec, Septentrion, 2010. Pp. 278. ISBN : 978-2-89448-638-2).}

\section{André-Yanne Parent}

Volume 33, numéro 2, 2011

URI : https://id.erudit.org/iderudit/1015035ar

DOI : https://doi.org/10.7202/1015035ar

Aller au sommaire du numéro

Éditeur(s)

Association Canadienne d'Ethnologie et de Folklore

ISSN

1481-5974 (imprimé)

1708-0401 (numérique)

Découvrir la revue

Citer ce compte rendu

Parent, A.-Y. (2011). Compte rendu de [Pierre Anctil et Ira Robinson (dirs.). Les communautés juives de Montréal, histoire et enjeux contemporains. (Québec, Septentrion, 2010. Pp. 278. ISBN : 978-2-89448-638-2).] Ethnologies, 33(2),

250-255. https://doi.org/10.7202/1015035ar d'utilisation que vous pouvez consulter en ligne.

https://apropos.erudit.org/fr/usagers/politique-dutilisation/ 


\section{Pierre Anctil et Ira Robinson (dirs.). Les communautés juives de Montréal, histoire et enjeux contemporains}

(Québec, Septentrion, 2010.

Pp. 278. ISBN : 978-2-89448-638-2).

\section{André-Yanne Parent Université de Montréal}

Les communautés juives de Montréal est l'un des rares ouvrages en français à combiner une analyse rigoureuse de l'apport de la communauté juive à la société montréalaise et, plus largement, québécoise, à l'étude poussée de l'influence de la société québécoise dans la création et le développement de l'identité juive montréalaise. La nature interdisciplinaire des différentes parties, qui se basent sur plusieurs méthodes d'analyses (histoire, littérature, sociologie, études religieuses, science politique), permet d'offrir un portrait qui rend justice aux multiples facettes de l'identité juive montréalaise, brisant ainsi les frontières disciplinaires.

Ces disciplines s'accordent toutes sur une conception non homogène de l'identité juive montréalaise, qui serait marquée par des rapports au judaïsme variés et des degrés d'influence changeants de la culture québécoise. Avec justesse, cet ouvrage présente les communautés juives montréalaises comme plurielles idéologiquement et culturellement.

Avec sa ferme volonté d'étayer le rapport historique et contemporain entre les communautés juives québécoises et le reste de la société pour mettre en valeur la contribution des premières dans l'évolution de la seconde et le caractère intrinsèquement relié des études juives et des études québécoises dans le contexte montréalais, l'ouvrage amène les chercheurs des études juives et des études québécoises à collaborer. En ce sens, Les communautés juives de Montréal se rapproche de la sociologie urbaine et démontre comment l'espace dans lequel un phénomène apparaît le façonne autant qu'il est inversement influencé par celui-ci. Composé d'une petite dizaine de parties, l'ouvrage se présente comme un excellent outil de vulgarisation de connaissances pointues, tout en offrant un portrait étoffé des communautés juives montréalaises. 
Respectant ce dessein de vulgarisation, les deux premières parties s'adressent surtout au lecteur néophyte. Ira Robinson réalise d'abord une synthèse efficace de l'histoire juive de l'ère ancienne à aujourd'hui. Si ce rapide survol apparaît à la fois ambitieux et immanquablement décevant pour les connaisseurs, ceux-ci ne peuvent que reconnaître le caractère probant du résultat qui, incontestablement, permet à celui qui découvre le sujet d'être bien outillé pour saisir les parties qui suivront. La deuxième partie, également d'Ira Robinson, poursuit cette mise en contexte en nous ramenant aux premiers jours du judaïsme à Montréal, à travers le parcours de ses premiers immigrants et le développement de ses premières synagogues. Les différents mouvements du judaïsme montréalais sont ainsi présentés, dont le trop souvent oublié mouvement reconstructionniste. En concluant ce portrait, l'auteur livre de premiers indices sur les spécificités du judaïsme montréalais : un niveau de pratique et d'affiliation institutionnelle plus élevé que chez la majorité des communautés juives d'Amérique du Nord, une croissance démographique soutenue, en partie liée à une forte présence hassidique, une importante communauté sépharade ou encore l'influence des communautés juives sur l'histoire et le développement de certains quartiers de la métropole québécoise.

La présence juive au Québec est également unique en Amérique du Nord du fait qu'elle se développe dans une société binaire, divisée entre les anglo-protestants et les franco-catholiques. Cette particularité est étudiée par Pierre Anctil dans une troisième partie assez instructive, qui retrace l'évolution de la relation entre les juifs montréalais et les francophones. L'auteur revient sur un sujet qui lui est cher et qu'il a souvent abordé dans plusieurs de ses ouvrages. Synthétique, il évoque comment le système d'éducation catholique a participé à créer ce « rendez-vous raté » entre les deux communautés, puis comment les premiers contacts se sont établis, notamment entre les ouvriers juifs et les ouvriers francophones, sans pour autant que cela empêche l'émergence d'un certain antisémitisme québécois. Il aura fallu attendre la Révolution tranquille, avec son mouvement de sécularisation progressive du Québec, pour que l'identité québécoise soit réfléchie moins en termes religieux que linguistiques, ce qui a participé largement au rapprochement des deux communautés. L'immigration massive de sépharades francophones a aussi donné un nouveau visage à l'identité juive montréalaise et, selon Pierre Anctil, 
a servi de pont entre les juifs déjà établis au Québec et la majorité francophone. Même si l'auteur relate que la commission BouchardTaylor a témoigné de la persistance de certains préjugés, surtout envers les Hassidim, cette partie se conclut sur une note positive, qui résume bien l'esprit de l'ouvrage : «En évitant de se cantonner dans des images traditionnelles et des rôles immuables, francophones et juifs ont ainsi ouvert un espace de négociation culturelle qui porte déjà ses fruits et qui permet une meilleure reconnaissance de l'apport de chacun dans la ville»(p. 62).

La quatrième partie réussit avec brio à présenter un élément crucial de l'histoire juive montréalaise : la place de la communauté dans l'espace scolaire québécois et les luttes quasi incessantes qui l'ont entourée, même au sein de la communauté juive, notamment entre les uptowners et les downtowners. L'auteur lève le voile sur l'origine des écoles privées juives, une histoire tout en rebondissements, où affrontements, négociations, accords et dissensions ont progressivement amené le système éducatif que l'on connaît aujourd'hui.

En quittant les bancs d'école, le lecteur est amené à voyager dans le Montréal yiddish avec Rebecca Margolis. Là encore, Montréal se distingue des villes analogues d'Amérique du Nord. Elle offre un espace privilégié pour la langue yiddish, laquelle, bien que moins parlée par les juifs montréalais, demeure très présente dans la vie culturelle de Montréal et dépasse les frontières communautaires. L'auteur nous amène également à comprendre comment la pratique du yiddish s'est transformée, avec la disparition progressive d'une génération de ses locuteurs, et s'est polarisée dans les dernières décennies en devenant à la fois une langue artistique séculière et une langue d'usage dans les communautés ultra-orthodoxes. Si le yiddish occupe une place centrale dans la culture musicale, littéraire et artistique montréalaise, Chantal Ringuet nous propose de dépasser les frontières linguistiques et de voir, de façon plus générale, «l'apport des créateurs juifs à la vie culturelle et littéraire montréalaise ». Cette partie détonne quelque peu du reste de l'ouvrage et apparaît davantage comme un catalogue qui passe en revue le parcours et les ouvres de plusieurs artistes juifs montréalais. Sans doute aurait-il été plus intéressant de brosser un tableau moins large, mais plus analytique des ouvres en veillant à les insérer dans le contexte social, politique et historique dans lequel elles sont apparues. 
La partie suivante évoque en profondeur comment les juifs ont contribué à la vie économique et syndicale montréalaise jusqu'à la Deuxième Guerre mondiale. Bernard Dansereau nous explique les conditions d'émergence d'un prolétariat typiquement juif, constitué de travailleurs des sweatshops et de nombreux artisans, et la façon dont ces ouvriers, majoritairement d'origine européenne, vont importer un modèle de militantisme qu'ils ont connu dans leur pays d'origine. Grâce à leur expérience européenne, les ouvriers juifs de la première moitié du XIX $\mathrm{X}^{\mathrm{e}}$ siècle vont être au cœur du mouvement syndical québécois, en plus de donner naissance à diverses organisations politiques. Cette partie, riche et détaillée, aborde une dimension souvent méconnue du syndicalisme québécois et témoigne de la contribution essentielle des ouvriers juifs à la société québécoise.

Sonia Zylberberg nous propose ensuite de nous concentrer sur les différentes expériences des femmes juives. Dans un style proche de l'essai, elle s'intéresse à celles qui, dans leur parcours, ont contribué à défendre la cause des femmes et à briser des frontières entre les genres. Les mouvements de solidarité comme les organisations bénévoles de femmes ont tous été animés par le dévouement ferme et continu d'individus engagés dans la promotion de la justice sociale et ont créé un terreau propice au développement des mouvements féministes qui ont suivi. Pour illustrer son propos, l'auteur, nous présente le portrait de quelques femmes d'exception, d'hier et d'aujourd'hui. Sonia Zylberberg parvient habilement à produire un tableau nuancé et pluriel de la place des femmes dans le judaïsme. Ce faisant, elle nous amène à réfléchir sur le caractère quelque peu tautologique d'un chapitre portant uniquement sur une telle catégorie, qui n'est pas, n'a jamais été et ne sera jamais homogène.

Cette partie nous fait basculer dans l'ère contemporaine. Une " analyse démographique et socioculturelle de la communauté juive montréalaise " lui succède. Basé à la fois sur des données quantitatives du recensement canadien de 2001 et sur des données plus qualitatives d'un sondage de la Fédération CJA, ce chapitre se caractérise par une rigueur empirique convaincante et enrichissante pour l'ensemble de l'ouvrage. Il permet de confirmer la diversité qui existe au sein des communautés juives montréalaises, la variété des modes d'expression de l'identité juive et le semblant de consensus au sein des communautés juives sur la qualité élevée de la vie juive à 
Montréal. Une seule critique subsiste toutefois, nous pouvons nous demander pourquoi cette partie arrive seulement à la fin de l'ouvrage. Si le respect de l'ordre chronologique des événements est le choix que les auteurs ont privilégié, nous aurions facilement pu imaginer cette partie juste à la suite des deux premières. Ce changement dans la structure de l'ouvrage participerait positivement à donner davantage de place et d'importance au contemporain.

L'ouvrage traite ensuite du sujet brûlant des communautés hassidiques de Montréal. Julien Bauer dépasse les préjugés et les fantasmes qui existent sur ces communautés avec ce portrait ancré historiquement et philosophiquement. Particulièrement instructif, surtout dans un contexte où ces communautés ont été largement caricaturées par les médias, ce chapitre nous amène à réfléchir non seulement aux différentes identités hassidiques, mais aussi à la vitalité de l'identité juive contemporaine et surtout à la " mosaïque » que constitue la société québécoise d'aujourd'hui et de demain, compte tenu de l'importante croissance démographique au sein de ces communautés ultra-orthodoxes.

La dernière partie retrace le parcours des juifs sépharades de Montréal et l'impact de leur présence à la fois dans la vie communauté juive et dans la relation entre les francophones et les communautés juives. Marqués par une expérience migratoire complexe, parfois vécue comme un exil forcé, et le dépaysement, les sépharades arrivent dans un contexte politique d'affirmation du fait français au Québec et de valorisation du multiculturalisme à la Chambre des communes. Au travers de ce que Yolande Cohen qualifie de " quête identitaire ", ces nouveaux arrivants aux traditions diverses vont négocier une identité inclusive, fondée sur une langue (le français) et une origine communes (simplifiée à l'Afrique du Nord). Cette communauté juive sépharade montréalaise apparaît donc comme une construction sociale, comme le suggère un segment du titre du chapitre, "comment devient-on sépharade ». Elle se distingue nettement des autres communautés juives de la ville tant en termes religieux, institutionnels et culturels que politiques. Ce dernier portrait participe autant à rendre compte de la diversité au sein des communautés juives montréalaises qu'à mettre en lumière les effets exceptionnels de la combinaison entre l'immigration sépharade et le contexte sociopolitique québécois dans lequel ces gens sont arrivés. 
La fin de l'ouvrage mise sur le fait que cette lecture veut attiser la curiosité des lecteurs et se présente comme une véritable invitation à continuer les recherches pour en découvrir davantage. Janice Rosen, du Service des archives du Congrès juif canadien, présente une liste descriptive et assez exhaustive des différentes ressources documentaires et archivistiques disponibles sur les juifs montréalais, dont l'utilité n'est pas à démontrer.

La principale critique qui peut être adressée à cet ouvrage est sa difficulté à aborder directement la dimension contemporaine. Si les mises en contexte historiques des premiers chapitres sont pertinentes et bien articulées, le contemporain n'est abordé que dans leurs dernières lignes. Les derniers chapitres de l'ouvrage lui accordent plus d'importance, mais là encore, la dimension historique supplante toujours l'analyse du contemporain. En outre, la structure chronologique de l'ouvrage accentue cet effet. Le livre aurait peut-être gagné à présenter une ethnographie d'une de ces communautés juives montréalaises afin de donner une importance première et sensible aux réalités juives d'aujourd'hui.

Ce livre est autant destiné à un large public qu'aux chercheurs, même si ces derniers risquent moins de découvrir un contenu « original». Il demeure néanmoins qu'aucun ouvrage en français ne rassemblait de la sorte autant de données sur les communautés juives montréalaises. Il convient aussi de souligner l'exceptionnel travail de synthèse sur lequel l'ouvrage repose.

La pertinence scientifique de cet ouvrage tient aussi au fait que la réalité juive québécoise, de surcroît montréalaise, est différente de l'expérience juive dans le reste du Canada. De fait, le contexte montréalais a permis le développement d'une identité juive unique et distincte, qui mérite l'attention particulière que Les communautés juives montréalaises lui porte. 